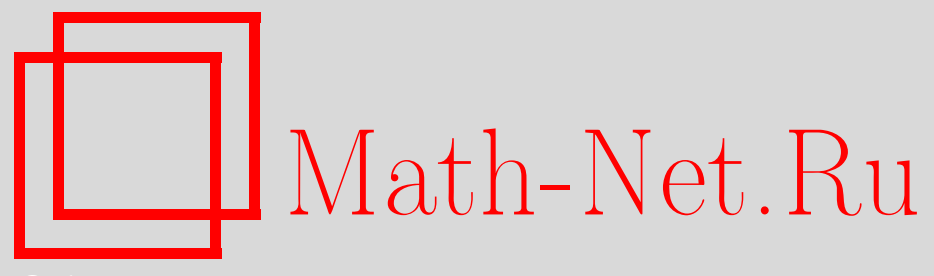

Н. В. Тимофеева, Компактификация в схеме Гильберта многообразия модулей стабильных 2-векторных расслоений на поверхности, Матем. заметки, 2007, том 82, выпуск 5, 756-769

DOI: https://doi.org/10.4213/mzm4087

Использование Общероссийского математического портала Math-Net.Ru подразумевает, что вы прочитали и согласны с пользовательским соглашением http: //www . mathnet.ru/rus/agreement

Параметры загрузки:

IP : 54.198 .67 .100

26 апреля 2023 г., 18:00:57

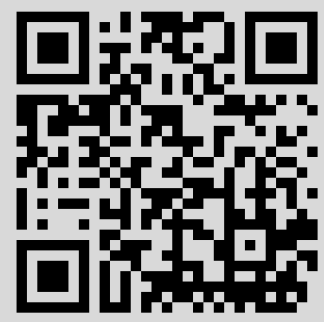


Том 82 выпуск 5 ноябрь 2007

УДК 512.7

\section{Компактификация в схеме Гильберта многообразия модулей стабильных 2-векторных расслоений на поверхности}

\section{Н. В. Тимофеева}

В статье построена новая компактификация многообразия модулей стабильных векторных 2-расслоений с классами Чженя $c_{1}, c_{2}$ для случая, когда универсальное семейство стабильных пучков с данными значениями инвариантов существует, а строго полустабильные пучки отсутствуют. Предложенная компактификация является подмногообразием в схеме Гильберта подсхем многообразия Грассмана, имеющих фиксированный многочлен Гильберта, и получается добавлением к многообразию модулей расслоений точек, соответствующих локально свободным пучкам на поверхностях, являющихся модификациями исходной поверхности. Кроме того, построен морфизм новой компактификации многообразия модулей на его компактификацию Гизекера-Маруямы.

Библиография: 8 названий.

Введение. В настоящей работе проведено построение новой компактификации тонкого многообразия модулей стабильных по Гизекеру [1] векторных расслоений ранга $r=2$ с классами Чженя $c_{1}, c_{2}$ на гладкой проективной алгебраической поверхности $S$ над алгебраически замкнутым полем характеристики 0 . Понятие стабильности по Гизекеру зависит от выбора поляризации $\mathrm{H} \in \mathrm{Pic} S$. Будем считать, что при поляризации $\mathrm{H}$ выполнено соотношение $G C D\left(r, c_{1} . \mathrm{H},(1 / 2) c_{1} \cdot\left(c_{1}-K_{S}\right)-c_{2}\right)=1$, где $K_{S}$ - канонический класс поверхности $S$, символ $G C D$ означает взятие наибольшего общего делителя. При этом $[2 ; 4.6 .7,4.6 .8]$ пространство модулей стабильных пучков является тонким, а его компактификация Гизекера-Маруямы $\bar{M}$ не содержит полустабильных, но не стабильных пучков. Тогда универсальное семейство $\mathbb{E}$ пучков, соответствующее пространству модулей стабильных пучков, определено на всем многообразии $\bar{M}$.

Во всем тексте данной работы используются следующие обозначения: $M_{0}-$ многообразие модулей стабильных 2-расслоений с классами Чженя $c_{1}, c_{2}$ на поверхности $S ; Y_{0}:=M_{0} \times S, Y:=\bar{M} \times S-$ произведения. Работа ведется в предположении, что $\bar{M}$ - неособое многообразие. В противном случае все рассуждения останутся в силе, если заменить особое многообразие $\bar{M}$ его гладким разрешением по Хиронаке [3].

В то время как компактификация Гизекера-Маруямы может содержать точки, соответствующие не локально свободным когерентным пучкам без кручения на поверхности $S$, предлагаемая в настоящей статье новая компактификация $\widetilde{M}$ состо- 
ит из точек, соответствующих локально свободным пучкам на поверхностях, являющихся модификациями поверхности $S$. Компактификация $\widetilde{M}$ является базой плоского семейства $\widetilde{Y} \stackrel{p_{1}}{\longrightarrow} \widetilde{M}$ двумерных подсхем некоторого многообразия Грассмана, имеющих в нем фиксированный многочлен Гильберта. Достаточно общий член семейства поверхностей $\widetilde{Y}$ изоморфен поверхности $S$. Многообразие $\widetilde{M}$ содержит открытое подмножество, изоморфное многообразию $M_{0}$, точки которого соответствуют расслоениям на поверхностях, изоморфных $S$, и обладает бирациональным морфизмом $\phi: \widetilde{M} \rightarrow \bar{M}$.

\section{1. Семейства пучков и гомологическая размерность пучка $\mathbb{E}$.}

ПреДЛОЖЕНИЕ 1. Пустъ $\mathscr{E}$ - плоское семейство стабилъных пучков без кручения на гладкой поверхности $S$, параметризованное равноразмерной схемой $T$. Тогда пучок Еீ обладает локально свободной резольвентой длины 1.

ДокАЗАтеЛЬСтво. Пусть $\mathscr{E} y-$ член семейства $\mathscr{E}$, соответствующий точке $y \in T$. По теореме о сизигиях пучок $\mathscr{E}_{y}$ обладает локально свободной резольвентой длины 2 :

$$
0 \rightarrow E_{2 y} \rightarrow E_{1 y} \rightarrow E_{0 y} \rightarrow \mathscr{E}_{y} \rightarrow 0
$$

Пусть $C_{1}=\operatorname{ker}\left(E_{0 y} \rightarrow \mathscr{E}_{y}\right)$. Дуализация точной последовательности (1) порождает две точные последовательности:

$$
\begin{gathered}
0 \rightarrow \mathscr{E}_{y}^{\vee} \rightarrow E_{0 y}^{\vee} \rightarrow C_{1}^{\vee} \rightarrow \operatorname{Ext}_{S}^{1}\left(\mathscr{E}_{y}, \mathscr{O}_{S}\right) \rightarrow 0, \\
0 \rightarrow C_{1}^{\vee} \rightarrow E_{1 y}^{\vee} \rightarrow E_{2 y}^{\vee} \rightarrow \operatorname{Ext}_{S}^{1}\left(C_{1}, \mathscr{O}_{S}\right) \rightarrow 0 .
\end{gathered}
$$

Введя обозначения $C_{2}=\operatorname{coker}\left(\mathscr{E}_{y}^{\vee} \rightarrow E_{0 y}^{\vee}\right), C_{3}=\operatorname{coker}\left(C_{1}^{\vee} \rightarrow E_{1 y}^{\vee}\right)$, имеем точные последовательности двойственных пучков

$$
\begin{gathered}
0 \rightarrow C_{2}^{\vee} \rightarrow E_{0 y} \rightarrow \mathscr{E}_{y}^{\vee \vee} \rightarrow \operatorname{Ext}_{S}^{1}\left(C_{2}, \mathscr{O}_{S}\right) \rightarrow 0, \\
0 \rightarrow C_{3}^{\vee} \rightarrow E_{1 y} \rightarrow C_{1}^{\vee \vee} \rightarrow \operatorname{Ext}_{S}^{1}\left(C_{3}, \mathscr{O}_{S}\right) \rightarrow 0
\end{gathered}
$$

Пучки $\mathscr{E}_{y}^{\vee \vee}$ и $C_{1}^{\vee \vee}$ локально свободны. Заметим, что по теореме о сизигиях пучок $C_{2}^{\vee}$ локально свободен, и, поскольку коразмерность подмножества особенностей пучка $\mathscr{E}_{y}$ равна 2, то $\operatorname{Ext}_{S}^{1}\left(\operatorname{Ext}_{S}^{1}\left(\mathscr{E}_{y}, \mathscr{O}_{S}\right), \mathscr{O}_{S}\right)=0$ и $C_{1}^{\vee \vee}=C_{2}^{\vee}$. Аналогично, в $(3)$ пучок $C_{3}^{\vee}$ локально свободен, $\operatorname{Ext}_{S}^{1}\left(\operatorname{Ext}_{S}^{1}\left(C_{1}^{\vee}, \mathscr{O}_{S}\right), \mathscr{O}_{S}\right)=0$ и $E_{2 y}=C_{3}^{\vee}$. Обозначив через $\mathscr{F}$ коядро канонического вложения $\mathscr{E}_{y} \hookrightarrow \mathscr{E}_{y} \vee \mathrm{V}$, имеем точную диаграмму (4).

По теореме о сизигиях пучок $\mathscr{F}$ имеет гомологическую размерность не более 2. Следовательно, пучок $\operatorname{ker}\left(E_{0 y} \rightarrow \mathscr{E}_{y}^{\vee \vee}\right)$ локально свободен. Следовательно, пучок $\mathscr{E} y$ обладает локально свободной резольвентой длины 1.

Для глобального случая достаточно заметить, что если семейство $\mathscr{E}$ не имеет локально свободной резольвенты длины меньше 2 , то найдется точка $y \in T$ такая, что пучок $\mathscr{E}_{y}$ обладает гомологической размерностью 2 , что противоречит доказанному.

ПреДЛОЖеНИЕ 2. Пучок $\mathscr{E}$ гомологической размерности 1 на гладкой схеме X, множество особенностей которого имеет коразмерность выше 2 , реблексивен. 


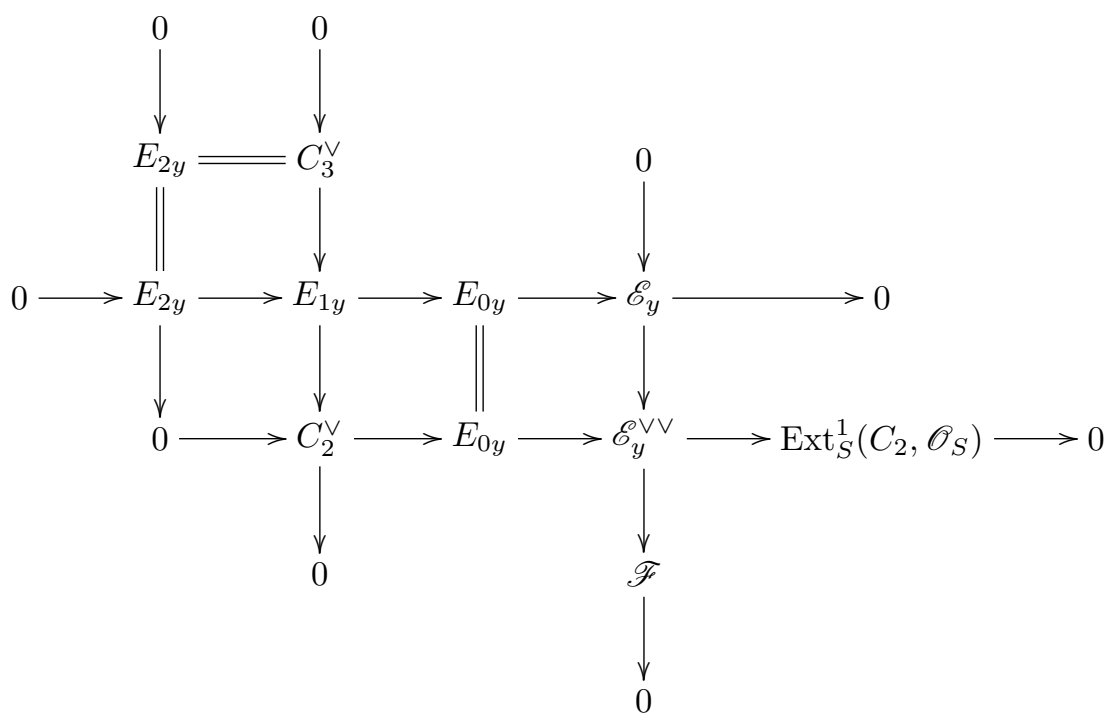

ДокАЗАТЕЛЬСтво. Пусть

$$
0 \rightarrow E_{1} \rightarrow E_{0} \rightarrow \mathscr{E} \rightarrow 0
$$

- локально свободная резольвента пучка $\mathscr{E}$; ее дуализация, обозначение $R=E_{0}^{\vee} / \mathscr{E} \vee$ и повторная дуализация приводят к точным последовательностям

$$
\begin{aligned}
& 0 \rightarrow R^{\vee} \rightarrow E_{0} \rightarrow \mathscr{E}^{\vee \vee} \rightarrow \operatorname{Ext}_{X}^{1}\left(R, \mathscr{O}_{X}\right) \rightarrow 0, \\
& 0 \rightarrow E_{1} \rightarrow R^{\vee} \rightarrow \operatorname{Ext}_{X}^{1}\left(\operatorname{Ext}_{X}^{1}\left(\mathscr{E}, \mathscr{O}_{X}\right), \mathscr{O}_{X}\right) \rightarrow 0, \\
& 0 \rightarrow \operatorname{Ext}_{X}^{1}\left(R, \mathscr{O}_{X}\right) \rightarrow \operatorname{Ext}_{X}^{2}\left(\operatorname{Ext}_{X}^{1}\left(\mathscr{E}, \mathscr{O}_{X}\right), \mathscr{O}_{X}\right) \rightarrow 0 .
\end{aligned}
$$

Так как $\operatorname{codim} \operatorname{Ext}_{\mathscr{O}_{X}}^{1}\left(\mathscr{E}, \mathscr{O}_{X}\right) \geqslant 3$, то

$$
\operatorname{Ext}_{\mathscr{O}_{X}}^{1}\left(\operatorname{Ext}_{X}^{1}\left(\mathscr{E}, \mathscr{O}_{X}\right), \mathscr{O}_{X}\right)=\operatorname{Ext}_{\mathscr{O}_{X}}^{2}\left(\operatorname{Ext}_{\mathscr{O}_{X}}^{1}\left(\mathscr{E}, \mathscr{O}_{X}\right), \mathscr{O}_{X}\right)=0
$$

и поэтому $\operatorname{Ext}_{X}^{1}\left(R, \mathscr{O}_{X}\right)=0, R^{\vee}=E_{1}$ и $\mathscr{E} \vee \vee=\mathscr{E}$.

Универсальное семейство пучков, соответствующее многообразию модулей стабильных пучков без кручения ранга 2 с классами Чженя $c_{1}, c_{2}$, удовлетворяет условиям обоих предложений, поэтому положим в $(5) \mathscr{E}=\mathbb{E}$. Далее пусть $\sigma: \widehat{Y} \rightarrow Y-$ морфизм раздутия схемы $Y$ в пучке идеалов Фиттинга $\mathscr{I}=\operatorname{Fitt}^{0}\left(\operatorname{Ext}_{Y}^{1}\left(\mathbb{E}, \mathscr{O}_{Y}\right)\right)$.

ПрЕДЛОЖЕниЕ 3. СХема $\widehat{Y}$ обладает локально свободным пучком Е ранга 2 таким, что $\left(\sigma_{*} E^{\vee}\right)^{\vee \vee}=\mathbb{E}$.

ДокАзАТЕЛЬСтво. Применение к (5) дуализации и функтора $\sigma^{*}$ приводит к точным последовательностям

$$
\begin{gathered}
\operatorname{Tor}_{1}^{\sigma^{-1} \mathscr{O}_{Y}}\left(\sigma^{-1} R, \mathscr{O}_{\widehat{Y}}\right) \rightarrow \sigma^{*} \mathbb{E}^{\vee} \rightarrow \sigma^{*} E_{0}^{\vee} \rightarrow \sigma^{*} R \rightarrow 0 \\
\operatorname{Tor}_{1}^{\sigma^{-1} \mathscr{O}_{Y}}\left(\sigma^{-1} \operatorname{Ext}_{\mathscr{O}_{Y}}^{1}\left(\mathbb{E}, \mathscr{O}_{Y}\right), \mathscr{O}_{\widehat{Y}}\right) \rightarrow \sigma^{*} R \rightarrow \sigma^{*} E_{1}^{\vee} \rightarrow \sigma^{*} \operatorname{Ext}_{\mathscr{O}_{Y}}^{1}\left(\mathbb{E}, \mathscr{O}_{Y}\right) \rightarrow 0 .
\end{gathered}
$$


Пусть в $(6 \mathrm{~b}) \mathscr{A}:=\operatorname{ker}\left(\sigma^{*} E_{1}^{\vee} \rightarrow \sigma^{*} \operatorname{Ext}_{\mathscr{O}_{Y}}^{1}\left(\mathbb{E}, \mathscr{O}_{Y}\right)\right)$. Рассмотрим пучок

$$
\operatorname{Fitt}^{0}\left(\sigma^{*} \operatorname{Ext}_{\mathscr{O}_{Y}}^{1}\left(\mathbb{E}, \mathscr{O}_{Y}\right)\right)
$$

Он обратим, так как

$$
\operatorname{Fitt}^{0}\left(\sigma^{*} \operatorname{Ext}_{\mathscr{O}_{Y}}^{1}\left(\mathbb{E}, \mathscr{O}_{Y}\right)\right)=\left(\sigma^{-1} \operatorname{Fitt}^{0}\left(\operatorname{Ext}_{\mathscr{O}_{Y}}^{1}\left(\mathbb{E}, \mathscr{O}_{Y}\right)\right)\right) \cdot \mathscr{O}_{\widehat{Y}}=\left(\sigma^{-1} \mathscr{I}\right) \cdot \mathscr{O}_{\widehat{Y}},
$$

где $Y$ - целая схема; последний пучок обратим по свойству раздутий. Теперь нам необходим следующий результат, принадлежащий Тихомирову.

ЛЕмма 4 [4; лемма 1]. Пусть $X$ - целая нетерова схема, и $\mathscr{F}$ - ненулевой ко-

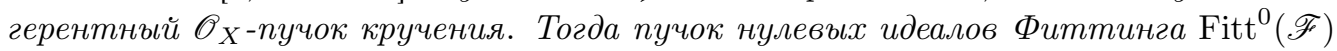
является обратимым $\mathscr{O}_{X}$-пучком тогда и только тогда, когда $\mathscr{F}$ имеет гомологическую размерность 1 , m.e. hd $\mathscr{F}=1$.

Тогда $\operatorname{hd} \sigma^{*} \operatorname{Ext}_{\mathscr{O}_{Y}}^{1}\left(\mathbb{E}, \mathscr{O}_{Y}\right)=1$. Таким образом, пучок $\mathscr{A}$ локально свободен, $\mathscr{A}=$ $\sigma^{*} R / \operatorname{tors}\left(\sigma^{*} R\right)$, где в правой части стоит факторпучок пучка $\sigma^{*} R$ по кручению, и $\mathscr{A}^{\vee}=\left(\sigma^{*} R\right)^{\vee}$.

Так как $\sigma^{*} E_{0}^{\vee}$ - пучок без кручения, то из (6а) следует точная тройка

$$
0 \rightarrow \sigma^{*} \mathbb{E}^{\vee} / \operatorname{tors}\left(\sigma^{*} \mathbb{E}^{\vee}\right) \rightarrow \sigma^{*} E_{0}^{\vee} \rightarrow \sigma^{*} R \rightarrow 0 .
$$

Пусть $E=\operatorname{ker}\left(\sigma^{*} E_{0}^{\vee} \rightarrow \mathscr{A}\right)$. Этот пучок локально свободен как ядро эпиморфизма локально свободных пучков. Принимая во внимание (7) и используя лемму о змее, получаем точную диаграмму

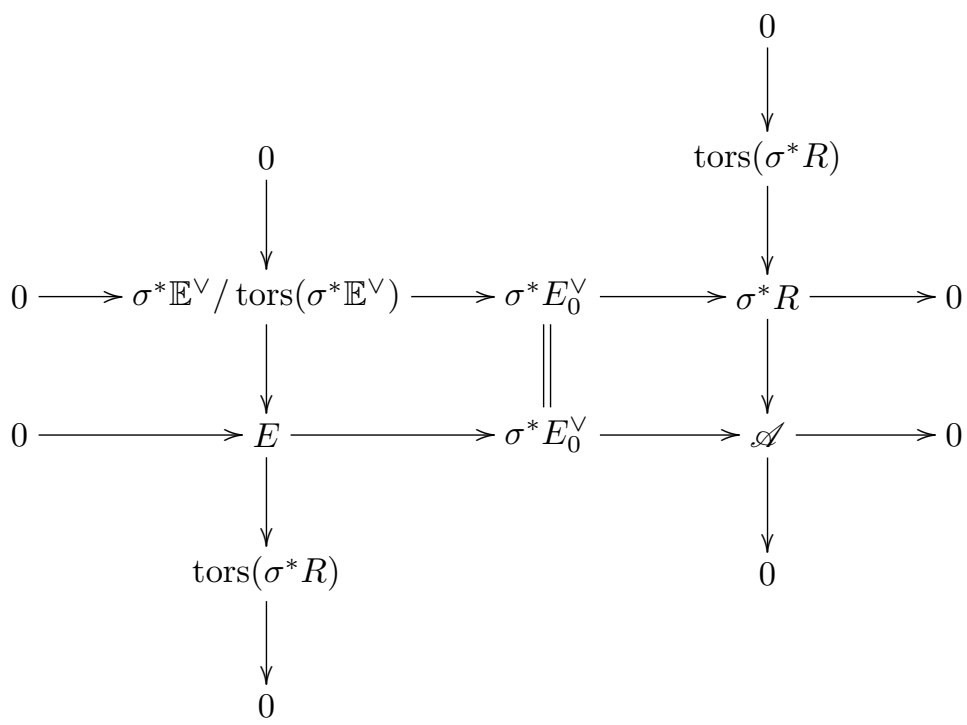

Дуализация левой вертикальной тройки в (8) и применение функтора $\sigma_{*}$ приводят к вложению $\sigma_{*} E^{\vee} \hookrightarrow \sigma_{*}\left(\sigma^{*} \mathbb{E}^{\vee}\right)^{\vee}$ пучков, совпадающих вне подмножества $Y \backslash Y_{0}$ коразмерности 3 . Образуя рефлексивные оболочки и учитывая рефлексивность пучка $\mathbb{E}$, имеем равенство $\left(\sigma_{*} E^{\vee}\right)^{\vee \vee}=\left(\sigma_{*}\left(\sigma^{*} \mathbb{E}^{\vee}\right)^{\vee}\right)^{\vee \vee}=\mathbb{E}$. 
2. Компактификация пространства $M_{0}$ и ее основные свойства. Зафиксируем основные обозначения и соглашения этого пункта:

- $\mathbb{E}_{0}:=\left.\mathbb{E}\right|_{Y_{0}}$

- $\widehat{\mathscr{L}}$ - очень обильный обратимый пучок на схеме $\widehat{Y}$;

- $G:=G(r, \mathrm{~V})$ - грассманиан подпространств размерности $r$ векторного пространства $\mathrm{V}$ размерности $\operatorname{dim} \mathrm{V}>r$; далее всюду $r=2$;

- $\mathscr{S}$ - тавтологическое подрасслоение ранга $r$ на многообразии $G$;

- $H:=\operatorname{Hilb}^{P(k)} G$ - схема Гильберта подсхем в многообразии Грассмана $G$, имеющих многочлен Гильберта, равный $P(k)$;

- $Z \subset H \times G$ - универсальное семейство подсхем над схемой Гильберта $H$;

- $p_{1}^{Z}: Z \rightarrow H, p_{2}^{Z}: Z \rightarrow G$ - проекции универсальной подсхемы $Z$.

Будут построены:

- $\widetilde{h}: \widetilde{M} \hookrightarrow H-$ замкнутое вложение новой компактификации $\widetilde{M}$ в схему Гильберта;

- $h_{0}=\left.\widetilde{h}\right|_{M_{0}}: M_{0} \hookrightarrow H-$ вложение открытого подмножества $M_{0} \subset \widetilde{M}$;

- $\widetilde{Y}:=\left(p_{1}^{Z}\right)^{-1} \widetilde{h}(\widetilde{M})$ - ограничение универсального семейства подсхем $Z$ на подсхему $\widetilde{M}$ в $H$;

- $\widetilde{h}_{Y}: \widetilde{Y} \hookrightarrow Z$ - вложение подсхемы $\widetilde{Y}$, индуцированное вложением $\widetilde{h}$;

- $h_{Y}=\left.\widetilde{h}_{Y}\right|_{Y_{0}}: Y_{0} \hookrightarrow Z$ - вложение открытого подмножества $Y_{0} \subset \tilde{Y}$;

- $p_{1}: \widetilde{Y} \rightarrow \widetilde{M}$ - структурная проекция семейства подсхем $\widetilde{Y}$;

- $j: \widehat{Y} \hookrightarrow G$ - замкнутое вложение;

- $j_{0}=\left.j\right|_{Y_{0}}: Y_{0} \hookrightarrow G$ - вложение открытого подмножества $Y_{0} \subset \widehat{Y}$, соответствующее вложению $j$;

- $p_{2}=\left.p_{2}^{Z}\right|_{\widehat{Y}}: \tilde{Y} \rightarrow \widehat{Y}$ - проекция, индуцированная проекцией $p_{2}^{Z}$;

- $\widetilde{\mathbb{E}}=\widetilde{h}_{Y}^{*}\left(\left.\mathscr{O}_{H} \otimes \mathscr{S}\right|_{Z}\right)$ - локально свободный пучок на схеме $\widetilde{Y}$;

- $\widehat{\Delta}:=\left(\operatorname{id}_{\widetilde{M}}, p_{2}\right)\left(p_{1} \times \operatorname{id}_{\widetilde{Y}}\right)(\widetilde{Y})-$ схемный образ морфизма $\widetilde{Y} \rightarrow \widetilde{M} \times \widehat{Y}$;

- $\Delta:=\left(\operatorname{id}_{\widetilde{M}}, \sigma \circ p_{2}\right)\left(p_{1} \times \operatorname{id}_{\widetilde{Y}}\right)(\widetilde{Y})-$ схемный образ морфизма $\widetilde{Y} \rightarrow \widetilde{M} \times Y$;

- $\widetilde{\pi}: \Delta \rightarrow \widetilde{M}-$ проекция, индуцированная вложением $\Delta \hookrightarrow \widetilde{M} \times Y$;

- $\phi: \widetilde{M} \rightarrow \bar{M}$ - морфизм новой компактификации на компактификацию Гизекера-Маруямы.

Пусть $\widehat{\mathscr{L}}$ - очень обильный обратимый пучок на $\widehat{Y}$. Выбором пучка $\widehat{\mathscr{L}}$ (например, заменив его при необходимости на достаточно большой степени кратный) можно добиться того, что

(i) пучок $\bigwedge^{2}\left(E^{\vee} \otimes \widehat{\mathscr{L}}\right)$ очень обилен;

(ii) естественное отображение $\bigwedge^{2} H^{0}\left(\widehat{Y}, E^{\vee} \otimes \widehat{\mathscr{L}}\right) \rightarrow H^{0}\left(\widehat{Y}, \bigwedge^{2}\left(E^{\vee} \otimes \widehat{\mathscr{L}}\right)\right)$ сюръективно.

Для (i) достаточно того, чтобы пучок $\bigwedge^{2}\left(E^{\vee} \otimes \widehat{\mathscr{L}}\right)=\left(\bigwedge^{2} E^{\vee}\right) \otimes \widehat{\mathscr{L}}^{\otimes 2}$ порождался глобальными сечениями; это гарантировано теоремой [5; II, теорема 5.17].

Возможность выбора $\widehat{\mathscr{L}}$ такого, что отображение

$$
\bigotimes^{2} H^{0}\left(\widehat{Y}, E^{\vee} \otimes \widehat{\mathscr{L}}\right) \rightarrow H^{0}\left(\widehat{Y}, \bigotimes^{2}\left(E^{\vee} \otimes \widehat{\mathscr{L}}\right)\right)
$$

сюръективно, дается в книге Лазарсфельда [6; пример 1.8.13]. Для перехода к внеш- 
нему квадрату рассмотрим очевидный эпиморфизм

$$
\bigotimes^{2} H^{0}\left(\widehat{Y}, E^{\vee} \otimes \widehat{\mathscr{L}}\right) \rightarrow \bigwedge^{2} H^{0}\left(\widehat{Y}, E^{\vee} \otimes \widehat{\mathscr{L}}\right)
$$

и цепочку равенств

$$
\begin{aligned}
H^{0}\left(\widehat{Y}, \bigotimes^{2}\left(E^{\vee} \otimes \widehat{\mathscr{L}}\right)\right) & =H^{0}\left(\widehat{Y}, \bigwedge^{2}\left(E^{\vee} \otimes \widehat{\mathscr{L}}\right) \oplus S^{2}\left(E^{\vee} \otimes \widehat{\mathscr{L}}\right)\right) \\
& =H^{0}\left(\widehat{Y}, \bigwedge^{2}\left(E^{\vee} \otimes \widehat{\mathscr{L}}\right)\right) \oplus H^{0}\left(\widehat{Y}, S^{2}\left(E^{\vee} \otimes \widehat{\mathscr{L}}\right)\right),
\end{aligned}
$$

приводящую к эпиморфизму проекции на прямое слагаемое

$$
H^{0}\left(\widehat{Y}, \stackrel{2}{\bigotimes}\left(E^{\vee} \otimes \widehat{\mathscr{L}}\right)\right) \rightarrow H^{0}\left(\widehat{Y}, \bigwedge^{2}\left(E^{\vee} \otimes \widehat{\mathscr{L}}\right)\right)
$$

Имеем коммутативную диаграмму

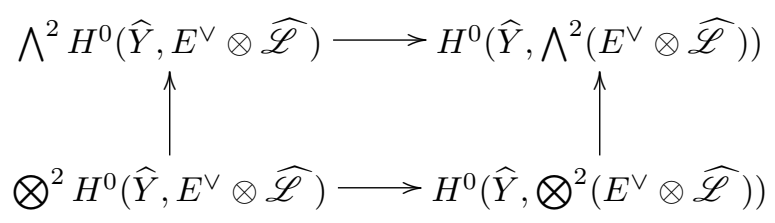

в которой известно, что все отображения кроме верхнего - эпиморфизмы. Тогда и верхняя стрелка - эпиморфизм. Теперь нам необходим следующий результат Траутманна (не опубликован).

Лемма 5. Пусть $\mathfrak{E}$ - локально свободный пучок ранга $r$ на многообразии $X, u$ nycms

(i) пучок $\bigwedge^{r} \mathfrak{E}$ очень обилен;

(ii) естественное отображение $\bigwedge^{r} H^{0}(X, \mathfrak{E}) \rightarrow H^{0}\left(X, \bigwedge^{r} \mathfrak{E}\right)$ сюргективно.

Тогда пучок $\mathfrak{E}$ порожден глобальными сечениями и ассоциированныи морфизм $\Phi_{\mathfrak{E}}$ : $X \rightarrow G\left(r, H^{0}(X, \mathfrak{E})^{\vee}\right)$ является замкнутым вложением.

ДокАЗАТЕЛЬСтво. По предположениям леммы отображение

$$
\bigwedge^{r} H^{0}(X, \mathfrak{E}) \otimes \mathscr{O}_{X} \rightarrow \bigwedge^{r} \mathfrak{E}
$$

сюръективно и, следовательно, отображение $H^{0}(X, \mathfrak{E}) \otimes \mathscr{O}_{X} \rightarrow \mathfrak{E}$ также сюръективно. Получаем коммутативную диаграмму

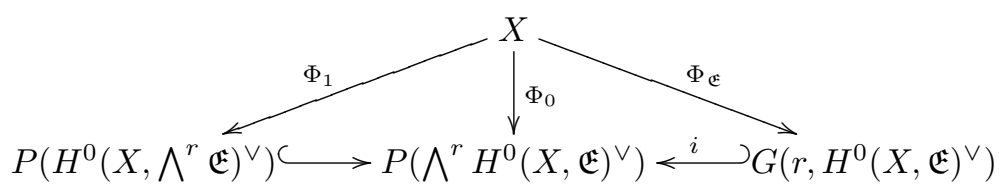

где $i$ - плюккерово вложение, $\Phi_{1}$ - замкнутое вложение, определенное очень обильным пучком $\bigwedge^{r} \mathfrak{E}, \Phi_{0}-$ морфизм, определенный сюръекцией (9). Тогда $\Phi_{0}-$ замкнутое вложение и согласно [5; II, упражнение 4.8 e] отображение $\Phi_{\mathfrak{E}}-$ замкнутое вложение. 
Теперь, полагая $\mathfrak{E}=E^{\vee} \otimes \widehat{\mathscr{L}}, r=2$, имеем индуцированное вложение $j: \widehat{Y} \hookrightarrow G$. Пусть $\mathscr{S}$ - тавтологическое подрасслоение ранга 2 на многообразии $G$. Вложением $j$ индуцировано равенство $E^{\vee} \otimes \widehat{\mathscr{L}}=j^{*} \mathscr{S}$.

Заметим, что согласно предложению 3 имеет место равенство $\mathbb{E}_{0}=\left.E^{\vee}\right|_{Y_{0}}$. Для открытого в $\widehat{Y}$ подмножества $Y_{0} \subset \widehat{Y}$ имеет место (индуцированное вложением $j$ ) вложение $j_{0}: Y_{0} \hookrightarrow G$, так что $\left.\mathbb{E}_{0} \otimes \widehat{\mathscr{L}}\right|_{Y_{0}}=j_{0}^{*} \mathscr{S}$.

Пусть $\mathscr{O}_{G}(1)$ - положительная образующая в группе Пикара многообразия Грассмана $G$. Многочлен Гильберта $P(k):=\chi\left(\left.j^{*} \mathscr{O}_{G}(k)\right|_{\{y\} \times S}\right)$ образа слоя $\{y\} \times S \subset Y_{0}$ при вложении $j_{0}$ не зависит от точки $y \in M_{0}$. Пусть $Z$ - универсальная подсхема в произведении $H \times G, p_{1}^{Z}: Z \rightarrow H$ - естественная проекция; тогда ввиду гладкости морфизма $\left.\pi\right|_{M_{0}}: Y_{0} \rightarrow M_{0}$ имеет место сохраняющее слои вложение плоского над базой $M_{0}$ семейства поверхностей $Y_{0}$ в универсальное (плоское над схемой Гильберта $H$ ) семейство подсхем $Z: Y_{0} \stackrel{h_{Y}}{\longleftrightarrow} Z$. Тогда определено индуцированное вложение базы $h_{0}: M_{0} \hookrightarrow H$ многообразия модулей стабильных векторных расслоений в схему Гильберта подсхем многообразия $G$, имеющих многочлен Гильберта $P(k)$, и имеет место диаграмма

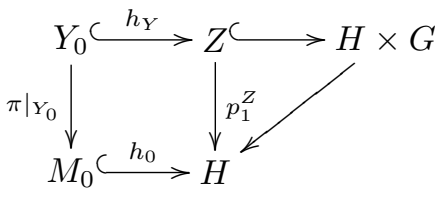

с расслоенным квадратом.

Образуем замыкание $\widetilde{M}$ образа $h_{0}\left(M_{0}\right) \cong M_{0}$ вложения $h_{0}$ и определим схему $\widetilde{Y} \supset Y_{0}$ с помощью расслоенной диаграммы

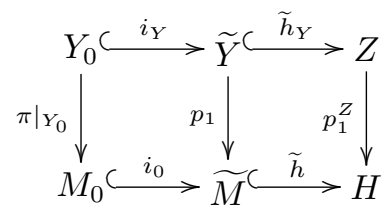

в которой $i_{0}, i_{Y}$ - открытые вложения и $\widetilde{h} \circ i_{0}=h_{0}, \widetilde{h}_{Y} \circ i_{Y}=h_{Y}$. Схема $\widetilde{Y}$ снабжена локально свободным пучком $\widetilde{\mathbb{E}}=\widetilde{h}_{Y}^{*}\left(\left.\mathscr{O}_{H} \otimes \mathscr{S}\right|_{Z}\right)$.

Для справедливости диаграммы

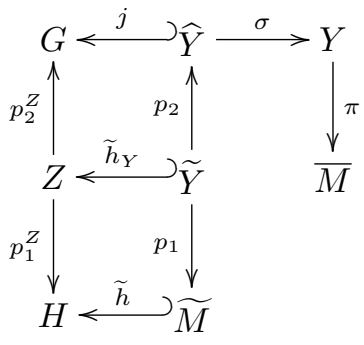

необходимо лишь убедиться в том, что $p_{2}^{Z}(\widetilde{Y})=\widehat{Y}$.

УТВЕРЖДЕНИЕ 6. Равенство верно на открытом подмножестве $Y_{0} \subset \widetilde{Y}$ :

$$
p_{2}^{Z}\left(h_{Y}\left(Y_{0}\right)\right)=j_{0}\left(Y_{0}\right) .
$$


ДокАзАтЕЛЬСтво. Морфизм $j_{0}$ осуществляет вложение произведения $Y_{0}=M_{0} \times S$ как семейства поверхностей, изоморфных $S$, в многообразие Грассмана с постоянным многочленом Гильберта $P(k)$. Пусть $\left(j_{0}\right)_{y}:\{y\} \times S \hookrightarrow G,\left(h_{Y}\right)_{y}:\{y\} \times S \hookrightarrow Z-$ вложения слоя $\{y\} \times S \subset Y_{0}$ над точкой $y \in M_{0}$. Морфизм $j_{0}$ индуцирует вложение $h_{Y}$ семейства $Y_{0}$ в универсальную подсхему $Z$ над схемой Гильберта, соответствующей многочлену Гильберта $P(k)$, определенное на слоях семейства $Y_{0}$ следующим образом:

$$
\left(h_{Y}\right)_{y}:\{y\} \times S \rightarrow\left(h_{y},\left(j_{0}\right)_{y}(\{y\} \times S)\right),
$$

где $h_{y}$ - точка схемы Гильберта $H$, соответствующая подсхеме $\left(j_{0}\right)_{y}(\{y\} \times S)$. Проекция $p_{2}^{Z}: Z \rightarrow G$ отображает слой $\left(h_{Y}\right)_{y}(\{y\} \times S)$ на образ слоя $\left(j_{0}\right)_{y}(\{y\} \times S)$. Таким образом, проекция $p_{2}^{Z}$ осуществляет соответствие образов семейства $Y_{0}$ : $p_{2}^{Z}\left(h_{Y}\left(Y_{0}\right)\right)=j_{0}\left(Y_{0}\right) \cong Y_{0}$. Утверждение доказано.

Образуем теоретико-схемное замыкание $\overline{h_{Y}\left(Y_{0}\right)}$.

УтвеРЖДЕНИЕ 7. Верно схемное равенство $\overline{h_{Y}\left(Y_{0}\right)}=\tilde{Y}$.

ДокАзАтЕЛЬство. Пусть $C_{0}$ - гладкая пунктированная кривая в $M_{0} \subset \widetilde{M}, C-$ ее схемное замыкание в $\widetilde{M}, p_{1}^{-1} C_{0} \subset Y_{0}$ - ее прообраз в $Z$. Тогда согласно [5; III, предложение 9.8] схемное замыкание $\overline{p_{1}^{-1} C_{0}}$ является плоской над $C$ подсхемой в схеме $Z$, причем слои схемы $\overline{p_{1}^{-1} C_{0}}$ и $\tilde{Y}$ над точками кривой $C$ совпадают, и $\overline{p_{1}^{-1} C_{0}}=p_{1}^{-1} C$. Таким образом, имеется композиция замкнутых вложений схем $p_{1}^{-1} C \hookrightarrow \overline{h_{Y}\left(Y_{0}\right)} \hookrightarrow \widetilde{Y}$, причем слои схемы $p_{1}^{-1} C$ совпадают с соответствующими слоями схемы $\widetilde{Y}$. Отсюда следует, что схемы $\overline{h_{Y}\left(Y_{0}\right)}$ и $\tilde{Y}$ совпадают послойно над $\widetilde{M}$. Пусть $\imath: \overline{h_{Y}\left(Y_{0}\right)} \hookrightarrow \widetilde{Y}-$ замкнутое вложение. Тогда имеется точная тройка $\mathscr{O}_{\widetilde{Y}}$-пучков

$$
0 \rightarrow \mathscr{K} \rightarrow \mathscr{O}_{\widetilde{Y}} \rightarrow \imath_{*} \mathscr{O}_{\overline{h_{Y}\left(Y_{0}\right)}} \rightarrow 0
$$

где пучок $\mathscr{K}$ определен как ядро эпиморфизма $\mathscr{O}_{\widetilde{Y}} \rightarrow \imath_{*} \mathscr{O}_{\overline{h_{Y}\left(Y_{0}\right)}}$. Так как схемы $\overline{h_{Y}\left(Y_{0}\right)}$ и $\widetilde{Y}$ совпадают послойно над $\widetilde{M}$, то многочлены Гильберта их слоев над $\widetilde{M}$ равны и схема $\overline{h_{Y}\left(Y_{0}\right)}$ является плоской над $\widetilde{M}$. Тогда оба пучка $\mathscr{O}_{\widetilde{Y}}$ и $\imath_{*} \mathscr{O}_{\overline{h_{Y}\left(Y_{0}\right)}}$ являются плоскими над $\widetilde{M}$. Следовательно, пучок ядра $\mathscr{K}$ плоский над $\widetilde{M}$, причем в общей точке его слой равен 0 . Значит, $\mathscr{K}=0$ и $\mathscr{O}_{\widetilde{Y}}=\imath_{*} \mathscr{O}_{\overline{h_{Y}\left(Y_{0}\right)}}, \imath-$ изоморфизм, что и требовалось доказать.

Очевидно, $\overline{j_{0}\left(Y_{0}\right)} \hookrightarrow \widehat{Y}$, и в силу собственности морфизма $p_{2}^{Z}$ имеется замкнутое вложение $p_{2}^{Z}(\widetilde{Y}) \hookrightarrow \widehat{Y}$, где $p_{2}^{Z}(\widetilde{Y})$ - схемный образ морфизма $p_{2}$. Так как схема $\widetilde{Y}$ приведена, то схемная структура на $p_{2}^{Z}(\tilde{Y})$ - приведенная структура [5; II, упражнение $3.11 \mathrm{~d}]$. Схема $\widehat{Y}$ приведена и неприводима как раздутие целой схемы [7; В.6.4], и $p_{2}^{Z}\left(Y_{0}\right)=j_{0}\left(Y_{0}\right)$, поэтому $\widehat{Y}=\overline{j_{0}\left(Y_{0}\right)}=p_{2}^{Z}(\tilde{Y})$.

Для дальнейшего потребуется

УтВеРЖДЕНИЕ 8. Справедливы равенства

$$
\widetilde{Y}=(\widetilde{h}(\widetilde{M}) \times G) \cap Z=(\widetilde{h}, j)(\widetilde{M} \times \widehat{Y}) \cap Z,
$$

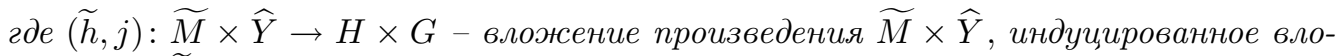
жениями $\widetilde{h}$ и 
ДокАзАТЕЛЬСтво. Первое равенство непосредственно следует из того, что универсальное семейство подсхем $Z$ является плоским над схемой Гильберта $H$, а $\widetilde{Y}-$ его ограничение на подсхему $\widetilde{M} \subset H$. Второе равенство получается из первого при учете равенства $p_{2}^{Z}(\widetilde{Y})=\widehat{Y}$.

ПрЕДЛОЖЕНИЕ 9. Определен регулярный бирачионалъный морфизм компактификаций $\phi: \widetilde{M} \rightarrow \bar{M}$.

ДокАЗАтЕЛЬСтво. Шаг 1. Отображение множеств. Положим по определению $\phi:=\left(\pi \circ \sigma \circ p_{2}\right) p_{1}^{-1}$. Для проверки корректности определения достаточно убедиться в том, что проекция $p_{2}$ сохраняет слои. Это означает, что слой проекции $p_{1}$ при морфизме $p_{2}$ переходит в слой композиции $\pi \circ \sigma$.

Рассмотрим открытое подмножество $M_{0} \subset \widetilde{M}$, точки которого соответствуют локально свободным пучкам. Подмножество $M_{0}$ порождает в универсальной подсхеме $Z$ подсемейство $h_{Y}\left(Y_{0}\right) \subset \widetilde{Y} \subset Z$, изоморфное $M_{0} \times S$. Согласно утверждению 6 $p_{2}\left(h_{Y}\left(Y_{0}\right)\right)=j_{0}\left(Y_{0}\right)$, причем по конструкции проекция $p_{2}$ на подмножестве $h_{Y}\left(Y_{0}\right)$ сохраняет слои над $M_{0}$.

Далее, пусть $\widetilde{y} \in \widetilde{M} \backslash h_{0}\left(M_{0}\right)$. Рассмотрим в $M_{0}$ гладкую кривую $C_{0}$ такую, что $\overline{h_{0}\left(C_{0}\right)} \backslash h_{0}\left(C_{0}\right)=\widetilde{y}$. В силу плоскости морфизма $p_{1}$ и универсальности схемы $Z$, $p_{1}^{-1} \overline{h_{0}\left(C_{0}\right)}=\overline{p_{1}^{-1}\left(h_{0}\left(C_{0}\right)\right)}$, слой семейства $p_{1}^{-1} \overline{h_{0}\left(C_{0}\right)}$ над точкой $\widetilde{y}$ совпадает со слоем $p_{1}^{-1}(\widetilde{y})$ и не зависит от выбора кривой $C_{0}$.

Рассмотрим вложение $l_{0}: C_{0} \hookrightarrow M_{0} \subset \bar{M}$ кривой $C_{0}$ такое, что проекция $p_{2}$ осуществляет сохраняющий слои изоморфизм локально замкнутых подсхем $p_{1}^{-1} C_{0} \subset \widetilde{Y}$ и $\sigma^{-1} \pi^{-1} l_{0}\left(C_{0}\right)$, расслоенных над $C_{0}$ и $l_{0}\left(C_{0}\right)$ соответственно. Образуя замыкания, имеем индуцированный проекцией $p_{2}$ бирациональный морфизм замкнутых подсхем $\overline{p_{1}^{-1}\left(C_{0}\right)} \rightarrow \overline{\sigma^{-1} \pi^{-1} l_{0}\left(C_{0}\right)}$. Пусть $\widetilde{y}=C \backslash C_{0} \in \widetilde{M}, \bar{y}=\overline{l_{0}\left(C_{0}\right)} \backslash l_{0}\left(C_{0}\right)$. Слой семейства $\widetilde{Y}$ над точкой $\widetilde{y}$ - поверхность, слой семейства $\frac{\bar{\sigma}}{\sigma^{-1} \pi^{-1} l_{0}\left(C_{0}\right)}$ над точкой $\bar{y}$ также поверхность по построению. Следовательно, проекция $p_{2}$ отображает слой $p_{1}^{-1}(\widetilde{y})$ семейства $\widetilde{Y}$ в слой схемы $\overline{\sigma^{-1} \pi^{-1} l_{0}\left(C_{0}\right)}$ над точкой $\bar{y}$.

Далее будем пользоваться обозначением $f=\pi \circ \sigma \circ p_{2}$.

Шаг 2. Непрерывное отображение топологических пространств. Для этого достаточно показать, что прообраз замкнутого по Зарисскому множества при отображении $\phi$ замкнут. В самом деле, композиция $f: \widetilde{Y} \rightarrow \bar{M}$ - настоящий морфизм, и поэтому непрерывна в топологии Зарисского. Пусть $A \subset \bar{M}$ - замкнутое по Зарисскому подмножество в схеме $\bar{M}$; тогда его прообраз $f^{-1}(A)$ замкнут в схеме $\widetilde{Y}$. Морфизм $p_{1}$ собственный и, следовательно, образ $p_{1} f^{-1}(A)$ замкнутого множества замкнут. По определению отображения $\phi$ прообраз замкнутого подмножества $A$ при этом отображении равен $\phi^{-1}(A)=p_{1} f^{-1}(A)$ и замкнут.

Шаг 3. Морфизм алгебраических схем. Необходимо построить морфизм пучков $\phi^{\sharp}: \mathscr{O}_{\bar{M}} \rightarrow \phi_{*} \mathscr{O}_{\widetilde{M}}$. Имеется морфизм пучков $f^{\sharp}: \mathscr{O}_{\bar{M}} \rightarrow f_{*} \mathscr{O}_{\widetilde{Y}}$. Очевидно, $\mathscr{O}_{\widetilde{Y}}=$ $p_{1}^{*} \mathscr{O}_{\widetilde{M}}$.

Проверим равенство функторов

$$
\phi_{*}=f_{*} p_{1}^{-1} .
$$

Пусть $\mathscr{F}$ - произвольный пучок на схеме $\widetilde{M}, V \subset \bar{M}$ - открытое подмножество. Согласно определению прямого образа пучка при непрерывном отображении топо- 
логических пространств $\left(\phi_{*} \mathscr{F}\right)(V)=\mathscr{F}\left(p_{1} f^{-1}(V)\right)$. Убедимся в том, что подмножество $W_{0}:=p_{1} f^{-1}(V)$ открыто в $\widetilde{M}$. Так как $V$ - открытое в $\bar{M}$ подмножество, то $A=\bar{M} \backslash V$ замкнуто. По доказанному (шаг 2) образ $p_{1} f^{-1}(A)$ замкнут. Очевидно, $p_{1} f^{-1}(A) \cup p_{1} f^{-1}(V)=\widetilde{M}$. Осталось проверить, что $p_{1} f^{-1}(A) \cap p_{1} f^{-1}(V)=\varnothing$. Предположив противное, выберем точку $\widetilde{y} \in p_{1} f^{-1}(A) \cap p_{1} f^{-1}(V)$. Тогда в слое $\widetilde{S}_{\widetilde{y}}=p_{1}^{-1}(\widetilde{y})$ найдутся точки $q_{1}$ и $q_{2}$ такие, что $p_{1}\left(q_{1}\right) \in p_{1} f^{-1}(A), p_{1}\left(q_{2}\right) \in p_{1} f^{-1}(V)$. Следовательно, в $\bar{M}$ найдутся точки $y_{1} \in A$ и $y_{2} \in V$ такие, что $f\left(q_{i}\right)=y_{i}, i=1,2$. Это противоречит корректности определения $\phi$ как отображения множеств. Отсюда следует, что $W_{0}:=p_{1} f^{-1}(V)$ открыто в $\widetilde{M}$ как дополнение к замкнутому множеству.

Далее обратимся к прообразу $\left(p_{1}^{-1} \mathscr{F}\right)(U)$, где $U:=f^{-1}(V)$. Согласно определению обратного образа пучка

$$
\left(p_{1}^{-1} \mathscr{F}\right)(U)=\lim _{W \supset W_{0}} \mathscr{F}(W)
$$

где справа стоит индуктивный предел по системе открытых подмножеств, содержащих образ $p_{1}(U)=W_{0}$.

Утверждается, что

$$
\lim _{W \supset W_{0}} \mathscr{F}(W)=\mathscr{F}\left(W_{0}\right)
$$

При проверке этого утверждения мы будем придерживаться следующих обозначений. Множества индуктивной системы - это $\mathscr{F}(W)$; они нумеруются открытыми подмножествами $W$. Для любых двух открытых множеств $W$ и $W^{\prime}$ таких, что $W^{\prime} \subset W$, определено отображение ограничения $f_{W^{\prime}}^{W}: \mathscr{F}(W) \rightarrow \mathscr{F}\left(W^{\prime}\right)$. Очевидно, что $f_{W}^{W}=\operatorname{id} \mathscr{F}(W)$ и $f_{W^{\prime}}^{W} \circ f_{W^{\prime \prime}}^{W^{\prime}}=f_{W^{\prime \prime}}^{W}$ для любых открытых подмножеств $W^{\prime \prime} \subset W^{\prime} \subset W$. Канонические отображения в индуктивный предел будем обозначать $f^{W}: \mathscr{F}(W) \rightarrow \lim _{W \supset W_{0}} \mathscr{F}(W)$ и $f^{W^{\prime}} \circ f_{W^{\prime}}^{W}=f^{W}$. Наконец, свойство универсальности индуктивного предела $[8 ; \mathrm{I}, 1.6]$ утверждает, что для множества $F$ и набора отображений $h^{W}: \mathscr{F}(W) \rightarrow F$ таких, что $h^{W^{\prime}}=h^{W} \circ f_{W}^{W^{\prime}}$, существует единственное отображение $h: \lim _{W \supset W_{0}} \mathscr{F}(W) \rightarrow F$ такое, что $h^{W}=h \circ f^{W}$.

Пара $\left(W_{0}, \mathscr{F}\left(W_{0}\right)\right)$ входит в рассматриваемую индуктивную систему. Поэтому имеется отображение $f^{W_{0}}: \mathscr{F}\left(W_{0}\right) \rightarrow \lim _{W \supset W_{0}} \mathscr{F}(W)$. Определены отображения ограничения $h^{W}=f_{W_{0}}^{W}: \mathscr{F}(W) \rightarrow \mathscr{F}\left(W_{0}\right)$ и по свойству универсальности индуктивного предела существует единственное отображение $h: \lim _{W \supset W_{0}} \mathscr{F}(W) \rightarrow \mathscr{F}\left(W_{0}\right)$.

Теперь рассмотрим диаграмму отображений

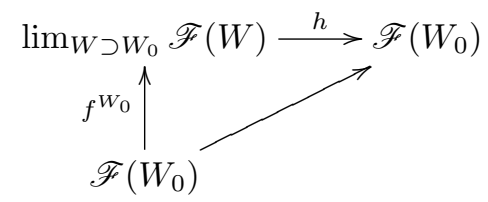

Здесь $f^{W_{0}}$ - отображение множества сечений $\mathscr{F}\left(W_{0}\right)$ как элемента индуктивной системы в индуктивный предел, $h$ - (единственное) отображение, индуцированное отображениями $h^{W_{0}}$ по универсальности индуктивного предела. Диагональное вложение - это отображение $h^{W_{0}}$, и по смыслу оно тождественно, и $h \circ f^{W_{0}}=\mathrm{id} \mathscr{F}\left(W_{0}\right)$. 
Теперь рассмотрим диаграмму

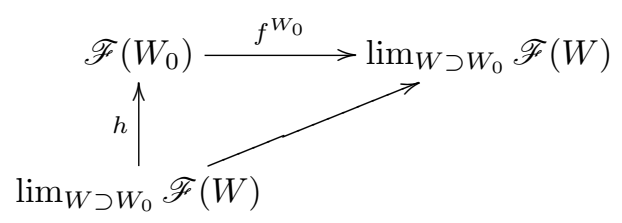

в которой диагональное отображение единственно в силу универсальности индуктивного предела. Следовательно, оно тождественно и $f^{W_{0}} \circ h=\operatorname{id}_{\lim _{W \supset W_{0}} \mathscr{F}(W)}$. Таким образом, $\mathscr{F}\left(W_{0}\right)=\lim _{W \supset W_{0}} \mathscr{F}(W)$.

Итак, $\left(p_{1}^{-1} \mathscr{F}\right)(U)=\mathscr{F}\left(p_{1}(U)\right)$. Вычислим сечения пучка $f_{*} p_{1}^{-1} \mathscr{F}$ :

$$
\left(f_{*} p_{1}^{-1} \mathscr{F}\right)(V)=\mathscr{F}\left(p_{1} f^{-1}(V)\right)=\left(\phi_{*} \mathscr{F}\right)(V) .
$$

Это доказывает равенство (10).

Тензорное умножение на $1^{\widetilde{Y}} \in \mathscr{O}_{\widetilde{Y}}$ определяет морфизм $\mathscr{O}_{\widetilde{M}}$-пучков

$$
\cdot \otimes_{p_{1}^{-1} \mathscr{O}_{\widetilde{M}}} 1^{\tilde{Y}}: p_{1}^{-1} \mathscr{O}_{\widetilde{M}} \rightarrow \mathscr{O}_{\widetilde{Y}}
$$

Он инъективен, так как $p_{1}$ - плоский морфизм схем. Применение функтора прямого образа $f_{*}$ приводит к мономорфизму $\rho:=f_{*}\left(\cdot \otimes_{p_{1}^{-1} \mathscr{O}_{\widetilde{M}}} 1^{\widetilde{Y}}\right): f_{*} p_{1}^{-1} \mathscr{O}_{\widetilde{M}} \rightarrow f_{*} \mathscr{O}_{\widetilde{Y}}$.

Пусть теперь $U$ - открытое в $\bar{M}$ подмножество; тогда морфизм пучков $f^{\sharp}: \mathscr{O}_{\bar{M}} \rightarrow$ $f_{*} \mathscr{O}_{\widetilde{Y}}$ порождает гомоморфизм колец $f^{\sharp}(U): \mathscr{O}_{\bar{M}}(U) \rightarrow\left(f_{*} \mathscr{O}_{\widetilde{Y}}\right)(U)=\mathscr{O}_{\widetilde{Y}}\left(f^{-1}(U)\right)$.

Далее, $\left(f_{*} p_{1}^{-1} \mathscr{O}_{\widetilde{M}}\right)(U)=p_{1}^{-1} \mathscr{O}_{\widetilde{M}}\left(f^{-1}(U)\right)=\mathscr{O}_{\widetilde{M}}\left(p_{1} f^{-1}(U)\right)=\mathscr{O}_{\widetilde{M}}\left(W_{0}\right)$, где $W_{0}=$ $p_{1} f^{-1}(U)$ - открытое в $\widetilde{M}$ подмножество.

Морфизм схем $p_{1}$ порождает гомоморфизм колец

$$
p_{1}^{\sharp}\left(W_{0}\right): \mathscr{O}_{\widetilde{M}}\left(W_{0}\right) \rightarrow\left(p_{1 *} \mathscr{O}_{\widetilde{Y}}\right)\left(W_{0}\right)=\mathscr{O}_{\widetilde{Y}}\left(p_{1}^{-1} p_{1} f^{-1}(U)\right) .
$$

Так как проекция $p_{2}$ переводит слой морфизма $p_{1}: \widetilde{Y} \rightarrow \widetilde{M}$ в слой композиции $\pi \circ \sigma$, то $p_{1}^{-1} p_{1} f^{-1}(U)=p_{1}^{-1} \phi^{-1}(U)=\left(\phi \circ p_{1}\right)^{-1}(U)=f^{-1}(U)$ и, таким образом, $\rho\left(W_{0}\right)=p_{1}^{\sharp}\left(W_{0}\right)$. Для произвольного сечения $s \in \mathscr{O}_{\widetilde{M}}\left(W_{0}\right) \rho(s)=s \cdot p_{1 *}\left(1^{\widetilde{Y}}\right)$.

Пусть $U_{0}=U \cap M_{0} \cong W_{0} \cap M_{0}$, и сечение $s^{\prime} \in \mathscr{O}_{\bar{M}}(U)$ таково, что ограничения $\left.s^{\prime}\right|_{U_{0}} \in \mathscr{O}_{\bar{M}}\left(U_{0}\right)$ и $\left.s\right|_{U_{0}} \in \mathscr{O}_{\widetilde{M}}\left(U_{0}\right)$ отождествляются при изоморфизме $\phi_{0}: \mathscr{O}_{\bar{M}}\left(U_{0}\right) \rightarrow$ $\mathscr{O}_{\widetilde{M}}\left(U_{0}\right): \phi_{0}\left(\left.s^{\prime}\right|_{U_{0}}\right)=\left.s\right|_{U_{0}}$. Тогда образ сечения $\left.s^{\prime}\right|_{U_{0}}$ при гомоморфизме $f^{\sharp}\left(U_{0}\right)$ равен $f^{\sharp}\left(\left.s^{\prime}\right|_{U_{0}}\right)=\left(\left.s^{\prime}\right|_{U_{0}}\right) \cdot f_{*}\left(1^{\tilde{Y}}\right)$.

Далее, $\rho \phi_{0}\left(\left.s^{\prime}\right|_{U_{0}}\right)=\rho\left(\left.s\right|_{U_{0}}\right)=\left(\left.s\right|_{U_{0}}\right) \cdot p_{1 *}\left(1^{\widetilde{Y}}\right)$ и справедливо равенство $\left(\left.s\right|_{U_{0}}\right)$. $p_{1 *}\left(1^{\widetilde{Y}}\right)=\left(\left.s^{\prime}\right|_{U_{0}}\right) \cdot f_{*}\left(1^{\widetilde{Y}}\right)$. Осталось убедиться в том, что это равенство можно продолжить до отображения $\phi^{\sharp}(U): \mathscr{O}_{\bar{M}}(U) \rightarrow \phi_{*} \mathscr{O}_{\widetilde{M}}(U)=\left(f_{*} p_{1}^{-1} \mathscr{O}_{\widetilde{M}}\right)(U)$, делающего коммутативной диаграмму

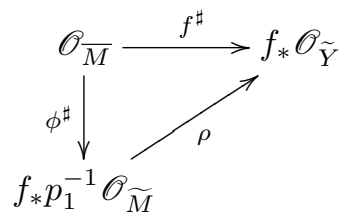


Для этого рассмотрим в $\bar{M}$ открытые подмножества $U_{1}$ и $U_{2}$ такие, что $U_{1} \cup U_{2}=U$, $U_{1} \cap U_{2}=U_{0}$, причем $s_{1} \in\left(\phi_{*} \mathscr{O}_{\widetilde{M}}\right)\left(U_{1}\right), s_{2} \in\left(\phi_{*} \mathscr{O}_{\widetilde{M}}\right)\left(U_{2}\right)$ и $s_{1} \cdot p_{1 *}\left(1^{\widetilde{Y}}\right)=\left(\left.s^{\prime}\right|_{U_{1}}\right) \cdot f_{*}\left(1^{\widetilde{Y}}\right)$, $s_{2} \cdot p_{1 *}\left(1^{\tilde{Y}}\right)=\left(\left.s^{\prime}\right|_{U_{2}}\right) \cdot f_{*}\left(1^{\tilde{Y}}\right)$. При этом $\left.s_{1}\right|_{U_{0}}=\left.s_{2}\right|_{U_{0}}$. Тогда по аксиоме пучка существует единственное сечение $s \in \mathscr{O}_{\bar{M}}(U)$ такое, что $\left.s\right|_{U_{1}}=\left.s_{1} s\right|_{U_{2}}=s_{2}$. Тогда $s=\phi^{\sharp}\left(s^{\prime}\right)$, что и требовалось.

Теперь рассмотрим сквозное отображение

$$
\left(\operatorname{id}_{\widetilde{M}}, \sigma \circ p_{2}\right): \widetilde{M} \times \widetilde{Y} \stackrel{\left(\mathrm{id}_{\widetilde{M}}, p_{2}\right)}{\longrightarrow} \widetilde{M} \times \widehat{Y} \stackrel{\left(\mathrm{id}_{\widetilde{M}}, \sigma\right)}{\longrightarrow} \widetilde{M} \times Y
$$

и вложение $\widetilde{Y} \stackrel{p_{1} \times \operatorname{id}_{\tilde{Y}}}{\hookrightarrow} \widetilde{M} \times \widetilde{Y}$, определяемое композицией $\widetilde{Y} \stackrel{\operatorname{diag}}{\longrightarrow} \widetilde{Y} \times \widetilde{Y} \stackrel{\left(p_{1}, \operatorname{id}_{\tilde{Y}}\right)}{\longrightarrow} \widetilde{M} \times \widetilde{Y}$ диагонального вложения и проекции первого сомножителя. Убедимся в том, что образ $\Delta:=\left(\operatorname{id}_{\widetilde{M}}, \sigma \circ p_{2}\right)\left(p_{1} \times \operatorname{id}_{\widetilde{Y}}\right)(\widetilde{Y})$ в произведении $\widetilde{M} \times Y$ изоморфен произведению $\widetilde{M} \times S$. Введем обозначения $\widetilde{\Delta}:=\left(p_{1} \times \operatorname{id}_{\widetilde{Y}}\right)(\widetilde{Y}), \widehat{\Delta}:=\left(\operatorname{id}_{\widetilde{M}}, p_{2}\right)(\widetilde{\Delta}) ;$ тогда $\Delta=$ $\left(\operatorname{id}_{\widetilde{M}}, \sigma\right)(\widehat{\Delta})$. Здесь $\widetilde{\Delta}, \widehat{\Delta}$ и $\Delta$ определяются как схемные образы соответствующих морфизмов. Схема $\widetilde{Y}$ приведена и неприводима, и схемный образ представляет собой приведенную структуру на замыкании ее образа, $\widetilde{\Delta} \cong \widetilde{Y}$. Тогда $\widehat{\Delta}$ и $\Delta-$ приведенные неприводимые схемы. В силу утверждения $8 \widehat{\Delta} \cong(\widetilde{h}, j)(\widetilde{M} \times \widehat{Y}) \cap Z=\widetilde{Y} \cong \widetilde{\Delta}$, т.е. $\left.\left(\operatorname{id}_{\widetilde{M}}, p_{2}\right)\right|_{\widetilde{\Delta}}-$ изоморфизм.

Рассмотрим диаграмму

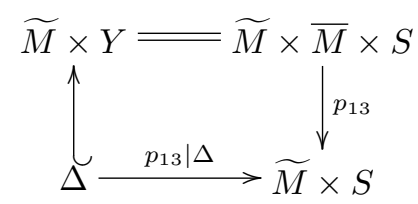

где $p_{13}$ - естественная проекция, и замкнутое вложение $\left(\left(\operatorname{id}_{\widetilde{M}} \times \phi\right), \operatorname{id}_{S}\right): \widetilde{M} \times S \rightarrow$ $\widetilde{M} \times Y$, индуцированное морфизмом $\phi$. По построению морфизма $\phi$ и схемы $\Delta$ как схемного образа $\left.\left(\operatorname{id}_{\widetilde{M}} \times \phi\right), \operatorname{id}_{S}\right)(\widetilde{M} \times S)=\Delta$. Отображение $p_{13} \mid \Delta$, очевидно, бирационально и по доказанному обладает двусторонним обратным, равным $\left(\left(\mathrm{id}_{\widetilde{M}} \times \phi\right), \mathrm{id}_{S}\right)$. Таким образом, $p_{13} \mid \Delta-$ изоморфизм.

Рассмотрим $\mathscr{O}_{Y}$-пучок $\left(\sigma_{*} \widehat{\mathscr{L}}\right)^{\vee}$. Он рефлексивен, имеет в общей точке ранг 1 и, следовательно, обратим. Пусть $\left(\sigma_{*} \widehat{\mathscr{L}}\right)^{\vee \vee}=\mathscr{O}_{Y}(D)$.

Теперь возьмем на $\widetilde{M} \times \widetilde{Y}$ локально свободный пучок $\mathscr{O}_{\widetilde{M}} \otimes \widetilde{\mathbb{E}} \otimes\left(\sigma \circ p_{2}\right)^{*} \mathscr{O}_{Y}(-D)$ и вычислим по формуле проекции его прямой образ при морфизме $\left(\mathrm{id}_{\widetilde{M}}, \sigma \circ p_{2}\right)$ :

$$
\left(\operatorname{id}_{\widetilde{M}}, \sigma \circ p_{2}\right)_{*}\left(\mathscr{O}_{\widetilde{M}} \otimes \widetilde{\mathbb{E}} \otimes\left(\sigma \circ p_{2}\right)^{*} \mathscr{O}_{Y}(-D)\right)=\mathscr{O}_{\widetilde{M}} \otimes\left(\sigma \circ p_{2}\right)_{*} \widetilde{\mathbb{E}} \otimes \mathscr{O}_{Y}(-D)
$$

В самом деле, формула проекции здесь применима, поскольку по теореме Зарисского $\left(\sigma \circ p_{2}\right)_{*} \mathscr{O}_{\widetilde{Y}}=\mathscr{O}_{Y}$. Используя явный вид пучка

$$
\widetilde{\mathbb{E}}=\widetilde{h}_{Y}^{*}\left(\left.\mathscr{O}_{H} \otimes \mathscr{S}\right|_{Z}\right)=p_{1}^{*} h^{*} \mathscr{O}_{H} \otimes_{\mathscr{O}_{\tilde{Y}}} p_{2}^{*} j^{*} \mathscr{S}=p_{1}^{*} \mathscr{O}_{\widetilde{M}} \otimes_{\mathscr{O}_{\tilde{Y}}} p_{2}^{*} j^{*} \mathscr{S}
$$

и соотношение $p_{1}^{*} \mathscr{O}_{\widetilde{M}}=\mathscr{O}_{\widetilde{Y}}$, получим

$$
\mathscr{O}_{\widetilde{M}} \otimes\left(\sigma \circ p_{2}\right)_{*} \widetilde{\mathbb{E}} \otimes \mathscr{O}_{Y}(-D)=\mathscr{O}_{\widetilde{M}} \otimes \sigma_{*} p_{2 *} p_{2}^{*} j^{*} \mathscr{S} \otimes \mathscr{O}_{Y}(-D)
$$


По формуле проекции имеем $\sigma_{*} p_{2 *} p_{2}^{*} j^{*} \mathscr{S}=\sigma_{*}\left(j^{*} \mathscr{S} \otimes p_{2 *} \mathscr{O}_{\widetilde{Y}}\right)$. Имеет место отображение вычисления $\sigma_{*} j^{*} \mathscr{S} \otimes \sigma_{*} p_{2 *} \mathscr{O}_{\widetilde{Y}} \rightarrow \sigma_{*}\left(j^{*} \mathscr{S} \otimes p_{2 *} \mathscr{O}_{\widetilde{Y}}\right)$. Стоящий слева пучок равен $\sigma_{*} j^{*} \mathscr{S}$. Применив равенство $E^{\vee} \otimes \widehat{\mathscr{L}}=j^{*} \mathscr{S}$ и тензорное умножение на $\bigotimes \mathscr{O}_{\widetilde{M}}$ и на $\mathscr{O}_{Y}(-D)$, имеем отображение

$$
\mathscr{O}_{\widetilde{M}} \otimes \sigma_{*}\left(E^{\vee} \otimes \widehat{\mathscr{L}}\right) \otimes \mathscr{O}_{Y}(-D) \rightarrow \mathscr{O}_{\widetilde{M}} \otimes \sigma_{*}\left(j^{*} \mathscr{S} \otimes p_{2 *} \mathscr{O}_{\widetilde{Y}}\right) \otimes \mathscr{O}_{Y}(-D)
$$

пучков одинакового ранга, совпадающих вне подмножества коразмерности 3. Тогда $\left(\sigma_{*}\left(E^{\vee} \otimes \widehat{\mathscr{L}}\right)\right)^{\vee \vee}=\left(\sigma_{*}\left(j^{*} \mathscr{S} \otimes p_{2 *} \mathscr{O}_{\widetilde{Y}}\right)\right)^{\vee \vee}$ и

$\left(\left(\operatorname{id}_{\widetilde{M}}, \sigma \circ p_{2}\right)_{*}\left(\mathscr{O}_{\widetilde{M}} \otimes \widetilde{\mathbb{E}} \otimes\left(\sigma \circ p_{2}\right)^{*} \mathscr{O}_{Y}(-D)\right)\right)^{\vee \vee}=\mathscr{O}_{\widetilde{M}} \otimes\left(\sigma_{*}\left(E^{\vee} \otimes \widehat{\mathscr{L}}\right)\right)^{\vee \vee} \otimes \mathscr{O}_{Y}(-D)$.

Рассмотрим морфизм $\sigma_{*} E^{\vee} \otimes \sigma_{*} \widehat{\mathscr{L}} \rightarrow \sigma_{*}\left(E^{\vee} \otimes \widehat{\mathscr{L}}\right)$ и связанный с ним морфизм рефлексивных оболочек $\left(\sigma_{*} E^{\vee} \otimes \sigma_{*} \widehat{\mathscr{L}}\right)^{\vee \vee} \rightarrow\left(\sigma_{*}\left(E^{\vee} \otimes \widehat{\mathscr{L}}\right)\right)^{\vee \vee}$. Когерентные рефлексивные пучки $\left(\sigma_{*} E^{\vee} \otimes \sigma_{*} \widehat{\mathscr{L}}\right)^{\vee \vee}$ и $\left(\sigma_{*}\left(E^{\vee} \otimes \widehat{\mathscr{L}}\right)\right)^{\vee \vee}$ на гладком комплексном многообразии $Y$ совпадают вне подмножества коразмерности 3. Следовательно, $\left(\sigma_{*} E^{\vee} \otimes \sigma_{*} \widehat{\mathscr{L}}\right)^{\vee \vee}=\left(\sigma_{*}\left(E^{\vee} \otimes \widehat{\mathscr{L}}\right)\right)^{\vee \vee}$.

Умножая канонический морфизм $\mu: \sigma_{*} \widehat{\mathscr{L}} \rightarrow\left(\sigma_{*} \widehat{\mathscr{L}}\right)^{\vee \vee}=\mathscr{O}_{Y}(D)$ тензорно на $\sigma_{*} E^{\vee}$, получим морфизм $\mathscr{O}_{Y}$-пучков $\mu_{E}: \sigma_{*} E^{\vee} \otimes \sigma_{*} \widehat{\mathscr{L}} \rightarrow \sigma_{*} E^{\vee} \otimes \mathscr{O}_{Y}(D)$. Тогда имеется индуцированное отображение дважды двойственных пучков

$$
\left(\mu_{E}\right)^{\vee \vee}:\left(\sigma_{*} E^{\vee} \otimes \sigma_{*} \widehat{\mathscr{L}}\right)^{\vee \vee} \rightarrow\left(\sigma_{*} E^{\vee} \otimes \mathscr{O}_{Y}(D)\right)^{\vee \vee} .
$$

Для пучка, стоящего справа, согласно предложению 3 справедливо равенство

$$
\left(\sigma_{*} E^{\vee} \otimes \mathscr{O}_{Y}(D)\right)^{\vee \vee}=\mathbb{E} \otimes \mathscr{O}_{Y}(D)=\mathbb{E}(D) .
$$

Итак, получено отображение $\left(\mu_{E}\right)^{\vee \vee}:\left(\sigma_{*} E^{\vee} \otimes \sigma_{*} \widehat{\mathscr{L}}\right)^{\vee \vee} \rightarrow \mathbb{E}(D)$ рефлексивных пучков, совпадающих вне подмножества коразмерности 3. Имеем равенство

$$
\left(\sigma_{*} E^{\vee} \otimes \sigma_{*} \widehat{\mathscr{L}}\right)^{\vee \vee}=\mathbb{E}(D) .
$$

Принимая во внимание изоморфизм $\Delta \cong \widetilde{M} \times S$ и выбрав произвольную точку $\widetilde{y} \in \widetilde{M}$, рассмотрим пучок

$$
\begin{aligned}
& \left.\left(\left.\left(\operatorname{id}_{\widetilde{M}}, \sigma \circ p_{2}\right)_{*}\left(\mathscr{O}_{\widetilde{M}} \otimes \widetilde{\mathbb{E}} \otimes\left(\sigma \circ p_{2}\right)^{*} \mathscr{O}_{Y}(-D)\right)^{\vee \vee}\right|_{\Delta}\right)\right|_{\{\widetilde{y}\} \times S}=\left.\left(\left.\left(\mathscr{O}_{\widetilde{M}} \otimes \mathbb{E}\right)\right|_{\Delta}\right)\right|_{\{\widetilde{y}\} \times S} \\
& \quad=k_{\widetilde{y}} \otimes\left(\left.\mathbb{E}\right|_{\{\phi(\widetilde{y})\} \times S}\right)=k_{\widetilde{y}} \otimes \mathscr{E}_{\phi(\widetilde{y})} .
\end{aligned}
$$

Таким образом, имеем явное описание морфизма $\phi: \widetilde{M} \rightarrow \bar{M}$ :

$$
\left.\widetilde{y} \mapsto\left(\left.\left(\left(\operatorname{id}_{\widetilde{M}}, \sigma \circ p_{2}\right)_{*}\left(\mathscr{O}_{\widetilde{M}} \otimes \widetilde{\mathbb{E}} \otimes\left(\sigma \circ p_{2}\right)^{*} \mathscr{O}_{Y}(-D)\right)\right)^{\vee \vee}\right|_{\Delta}\right)\right|_{\{\widetilde{y}\} \times S}
$$

Итак, доказана

ТЕОРемА. Для стабильных векторных расслоений ранга 2 на гладкой проективной алгебраической поверхности с классами Чженя $c_{1}, c_{2}$ существует компактибикация $\widetilde{M}$ тонкого пространства модулей вместе с ее вложением в схему Гильберта $H$ подсхем с фиксированным многочленом Гильберта в многообразии Грассмана $G$, включающаяся в диаграмму

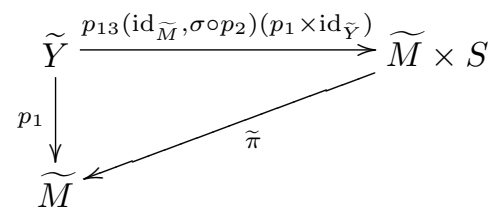


где $\widetilde{Y}$ - ограничение универсального семейства подсхем на $\widetilde{M} \subset H$. Морфизмы $\left(\operatorname{id}_{\widetilde{M}}, \sigma \circ p_{2}\right),\left(p_{1} \times \mathrm{id}_{\widetilde{Y}}\right)$ и $p_{13}$ определены в (11) и (12). Кроме того, имеется регулярный бирациональный морфизм новой компактификации на компактификацию Гизекера-Маруямы, определяемый выражением

$$
\phi: \widetilde{M} \rightarrow \bar{M}:\left.\widetilde{y} \mapsto\left(\left.\left(\left(\operatorname{id}_{\widetilde{M}}, \sigma \circ p_{2}\right)_{*}\left(\mathscr{O}_{\widetilde{M}} \otimes \widetilde{\mathbb{E}} \otimes\left(\sigma \circ p_{2}\right)^{*} \mathscr{O}_{Y}(-D)\right)\right)^{\vee \vee}\right|_{\Delta}\right)\right|_{\{\widetilde{y}\} \times S},
$$

в котором $\widetilde{\mathbb{E}}-$ локально свободный $\mathscr{O}_{\widetilde{Y}}$-пучок, $\Delta$ - подсхема в $\widetilde{M} \times Y$, изоморфная произведению $\widetilde{M} \times S, D$ - класс дивизоров на многообразии $Y$, не зависящий от точки $\widetilde{y} \in \widetilde{M}$. При этом диаграмма

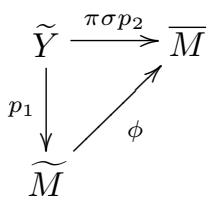

коммутативна.

\section{СПИСОК ЦИТИРОВАННОЙ ЛИТЕРАТУРЫ}

[1] D. Gieseker, "On the moduli of vector bundles on an algebraic surface", Ann. of Math. (2), 106:1 (1977), 45-60.

[2] D. Huybrechts, M. Lehn, Geometry of Moduli Spaces of Sheaves, Aspects of Mathematics, E31, Vieweg, Braunschweig, 1997.

[3] H. Hironaka, "Resolution of singularities of an algebraic variety over a field of characteristic zero. I.", Ann. of Math. (2), 79:1 (1964), 109-203.

[4] А.С. Тихомиров, "Многообразие полных пар нульмерных подсхем алгебраической поверхности", Изв. РАН. Сер. матем., 61:6 (1997), 153-180.

[5] Р. Хартсхорн, Алгебраическая геометрия, Мир, М., 1981.

[6] R. Lazarsfeld, Positivity in Algebraic Geometry. I., Classical setting: line bundles and linear series, Ergebnisse der Mathematik und Ihrer Grenzgebiete. 3 Folge A Series of Modern Surveys in Mathematics, 48, Springer-Verlag, Berlin, 2004.

[7] У. Фултон, Теория пересечений, Мир, М., 1989.

[8] Р. Годеман, Алгебраическая топология и теория пучков, ИЛ, М., 1961.

Н. В. Тимофеева

Ярославский государственный педагогический университет им. К. Д. Ушинского

E-mail: ntimofeeva@list.ru
Поступило

27.04.2006

Исправленный вариант

28.05.2007 